\title{
Site-directed mutagenesis of maize elite germplasm through pollination by cas9/gRNA-transgenic, haploidy-inducing lines
}

\author{
Daghma D.E.S.*, Mirzakhmedov M., Chamas S., Büchner H., Satpathy P., \\ Kumlehn J. \\ Leibniz. Institute of Plant Genetics and Crop Plant Research (IPK), Plant Reproductive Biology, \\ Gatersleben, Germany \\ *email:daghma@ipk-gatersleben.de
}

To fully exploit the immense potential of genome editing for plant breeding, it is necessary to induce target sequence-specific modifications directly using elite germplasm. With this goal in mind, we are pursuing a new strategy based on the utilization of haploidy-inducing maize lines. Such lines need to be transformed with cas 9 and guide (g)RNA expression units and then used as pollinators. Upon fertilization, the target motif in the hybrid zygote's maternal genome may be processed by the transgene products introduced from the sperm cell, whereas the transgene-bearing pollinator genome is being eliminated during early embryogenesis. Our concept further involves that the egg cell-derived genome remaining in the haploid embryo, including any Cas endonuclease-induced modification, can be identically duplicated by colchicine treatment. Outcome of this procedure will be progeny with edits in homozygous condition in the genetic background of the mother plant, while carrying neither portions of the haploidy-inducing pollinator line nor Cas9 and gRNA encoding transgenes. The possibility of directly editing elite lines that have hitherto not been amenable to transformation arises from the fact that haploidy-inducing lines can be utilized to pollinate virtually any maize accession of choice. However, the difficulty to transform available haploidy inducer lines, prompted us to generate new such lines via Cas9mediated knockout of the PHOSPHOLIPASE Al (PLA1/MTL/NLD) gene, which is known to confer haploid-inducing ability to maize. Accordingly, two transformation vectors carrying three $P L A l$-specific gRNAs were first functionally validated via PEGmediated transfection of maize protoplasts. Subsequently, Agrobacterium-mediated transformation of Hi-II maize was conducted. The analysis of the target motifs in primary transgenic plants revealed that 23 out of 24 and 26 out of 28 plants carried mutations in target motifs 1 and 3 respectively. The selection of homozygous mutants among progeny, their test for haploidy-inducing capability and retransformation with cas $9 / \mathrm{gRNA}$ constructs addressing diverse target genes of interest is in progress. 